\title{
Enhanced exciton transfer from the cascaded bilayer of green- and red-emitting CdTe quantum dots into bulk silicon
}

\author{
Aydan Yeltik ${ }^{1}$, Burak Guzelturk ${ }^{1}$, and Hilmi Volkan Demir ${ }^{1,2}$ \\ ${ }^{I}$ Department of Physics, Department of Electrical and Electronics Engineering, and UNAM - Institute of Materials Science and Nanotechnology \\ Bilkent University, Ankara, Turkey TR-06800 \\ ${ }^{2}$ LUMINOUS! Center of Excellence for Semiconductor Lighting and Displays, Microelectronics Division, School of Electrical and Electronics \\ Engineering, and Physics and Applied Physics Division, School of Physical and Mathematical Sciences, Nanyang Technological University, \\ Nanyang Avenue, Singapore 639798, Singapore \\ Email:volkan@bilkent.edu.tr and hvdemir@ntu.edu.sg
}

\begin{abstract}
We show enhanced transfer of excitons from the energy-gradient of bilayered green/red-emitting quantum dots into silicon using cascaded nonradiative energy with an overall enhancement factor of 1.3 at room temperature for solar cell sensitization. (C)2013 Optical Society of America

OCIS codes: (260.2160) Energy transfer; (160.4236) Nanomaterials; (040.6040) Silicon
\end{abstract}

Crystalline silicon is the most dominating material in today's photovoltaic industry. [1] However, silicon is limited in its material properties including optical absorption, which could be overcome via alternative approaches in Sibased photovoltaic devices. Among these, energy transfer based hybrid nanostructures stand out as a promising candidate owing to the increased potential utilization of the solar spectrum. [2,3] Previously, radiative energy transfer and nonradiative energy transfer (NRET) have been investigated by using colloidal quantum dots (QDs), while also being exploited as efficient exciton donors to silicon, in various hybrid nanostructures including bulk, nanopillar and nanomembrane silicon. [3-5] In these systems, a single type of QDs have been used for the purpose of energy conversion. Recently, cascaded energy conversion systems with more than one type of QDs have arised. [68] In this work, different from the previous studies, we proposed and demonstrated a hybrid construct of bilayered green- and red-emitting CdTe QDs on bulk Si to enhance the sensitization of Si by the energy-gradient QDs. Here the resulting NRET dynamics in the structure of such cascaded colloidal green- and red-emitting CdTe QDs on Si were investigated and compared to the structures with a single type of QDs.

In this work, we fabricated hybrid structures of bilayered CdTe QDs on p-type Si (100) with a controlled polyelectrolyte separation layer using layer-by-layer deposition technique. Fig. 1(a)-(c) illustrate the generic architecture of the working samples consisting of QD bilayers separated by consecutive adsorption of positively charged PDDA and negatively charged PSS polyelectrolytes. The Si substrate is $0.5 \mathrm{~mm}$ thick and possesses approximately $1.7 \mathrm{~nm}$ thick native oxide on the top, as verified by ellipsometer.

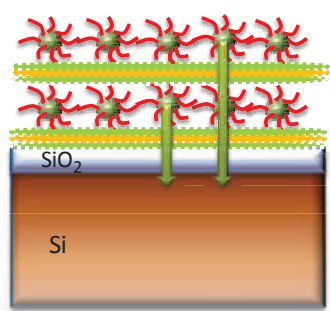

(a)

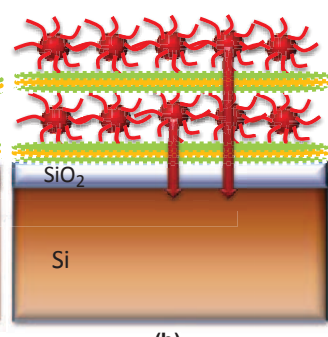

(b)

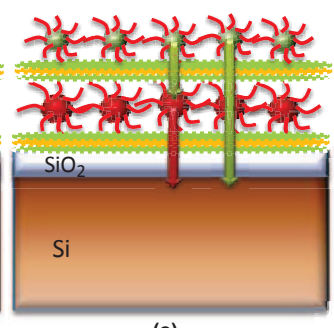

(c)

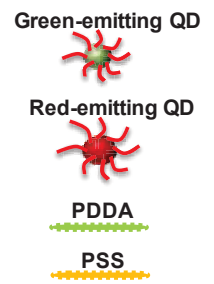

Fig. 1. Schematic for the energy transfer from the bilayer of (a) green-emitting QDs and (b) red-emitting QDs, and (c) the gradient energy transfer from the cascaded bilayer of green- and red-emitting QDs into bulk Si.

In Fig. 2(a), the emission and absorption spectra of green-emitting and red-emitting CdTe QDs (GQDs and RQDs, respectively), and the absorption spectrum of bulk Si are presented to illustrate the strong spectral overlap between the QDs and Si. We investigated the emission dynamics of GQDs and RQDs for the integrated bilayer QDbulk Si system at their peak emission wavelengths of 640 and $535 \mathrm{~nm}$, respectively. To assess the NRET dynamics, fluorescence decays of the QDs were recorded at room temperature by time resolved fluorescence (TRF) 
spectroscopy. We used the 1/e fitting for the decays of these QDs, and the extracted exciton lifetimes were presented in Fig. 2(b).

Fig. 2(c) shows the TRF decay curves of RQD bilayer-Si, G-R QD bilayer-Si, and their control groups on quartz substrate at the RQD emission peak. We observed faster decays for the cases of RQD bilayer-Si and G-R QD bilayer-Si as compared to their respective control groups on quartz. This is the indication of NRET from QDs into $\mathrm{Si}$. More importantly, after the integration of bilayer consisting of one top-monolayer GQD and one bottommonolayer RQD on $\mathrm{Si}$, the lifetime of RQDs was measured to be $0.71 \mathrm{~ns}$. This indicates the formation of energy gradient, since the lifetime for RQD bilayer-Si was measured to be $0.35 \mathrm{~ns}$.

We further investigated the exciton transfer dynamics of the donor QDs at the GQD emission peak to verify the gradient exciton feeding from the cascaded bilayer of different sized QDs into Si. TRF decay curves of GQD bilayer-Si, G-R QD bilayer-Si and their respective control groups on quartz substrates are given in Fig. 2(d). As expected from the previous results, all TRF lifetimes extracted for the QDs on quartz exceeded the lifetimes for the QDs on Si, which confirms the energy transfer from QDs into Si (see Fig. 2(b)). Moreover, after the integration of the bilayer consisting of one top-monolayer GQD and one bottom-monolayer RQD on $\mathrm{Si}$, the lifetime is shortened down to $0.26 \mathrm{~ns}$, while it was $0.36 \mathrm{~ns}$ for the GQDs bilayer-Si. Including the effects resulted from the differences in the quantum yields of the QDs and in the substrate refractive indices, we found the resulting overall enhancement factor of the exciton transfer into Si to be 1.3. This is an indication for the formation of gradient energy transfer, which leads to higher exciton population in RQDs, allowing for stronger NRET into $\mathrm{Si}$ as compared to non-gradient structures.

(a)

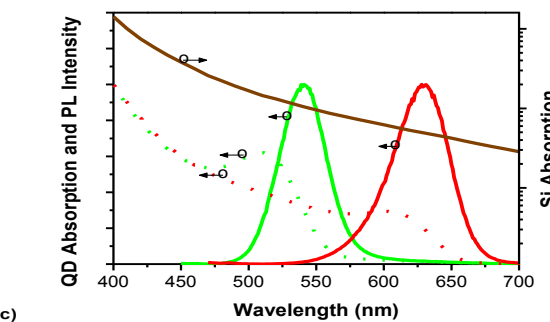

(c)

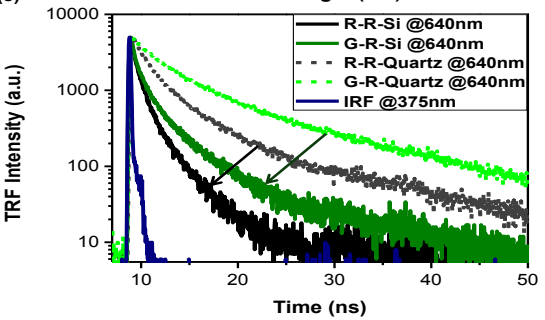

\begin{tabular}{|c|c|c|c|c|}
\hline \multirow{2}{*}{ Lifetime (ns) } & G-G QDs & R-R QDs & \multicolumn{2}{c|}{ G-R QDs } \\
\cline { 2 - 5 } & $535 \mathrm{~nm}$ & $640 \mathrm{~nm}$ & $535 \mathrm{~nm}$ & $640 \mathrm{~nm}$ \\
\hline Si & 0.363 & 0.350 & 0.263 & 0.707 \\
Quartz & 0.581 & 2.760 & 0.426 & 4.310 \\
\hline
\end{tabular}

(d)

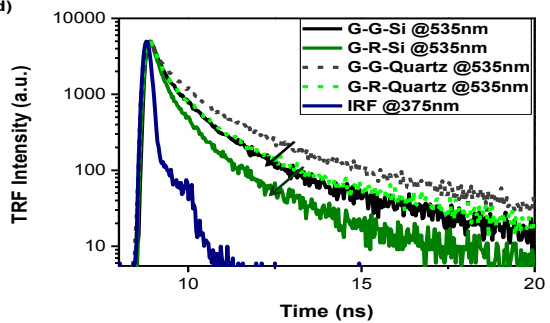

Fig. 2. (a) PL emission spectra of QDs (green and red solid lines for GQDs and RQDs, respectively), and absorption spectra of Si (brown solid line) and QDs (green and red dotted lines for GQDs and RQDs, respectively), (b) TRF lifetimes for all hybrid QD-QD-Si and QD-QD -Quartz structures used, (c) TRF decay curves at $640 \mathrm{~nm}$ from bilayer integration of R-R QDs on Si (black solid line), G-R QDs on Si (olive solid line), R-R QDs on quartz (dark-gray dashed line), G-R QDs on quartz (green dashed line), and the laser diode response function (navy solid line), (d) TRF decay curves at $535 \mathrm{~nm}$ from the bilayer integration of G-G QDs on Si (black solid line), G-R QDs on Si (olive solid line), G-G QDs on quartz (dark-gray dashed line), G-R QDs on quartz (green dashed line), and the laser diode response function (navy solid line).

In conclusion, we presented increased exciton transfer efficiency from CdTe QDs into Si through NRET by using the hybrid structures including a cascaded bilayer of multi-sized QDs on Si instead of non-cascaded structures. An enhanced exciton transfer into Si with an enhancement factor of 1.3 was achieved with the hybridization of energy gradient in the bilayered structures at room temperature, which is promising for more efficient Si-based solar cells in near future.

This work is supported in part by EU-FP7 Nanophotonics4Energy NoE. H.V.D. acknowledges support from ESF-EURYI and TUBAGEBIP; and A.Y. from TUBITAK BIDEB.

\section{References}

[1] A. Müller, M. Ghosha, R. Sonnenschein and P. Woditsch, Mater. Sci. Eng. B 134, 257-262 (2006).

[2] V. M. Agranovich, Y. N. Gartstein and M. Litinskaya, Chem. Rev. 111, 5179-5124 (2011).

[3] B. Guzelturk, E. Mutlugun, X. Wang, K. L. Pey and H. V. Demir, Appl. Phys. Lett. 97, 093111 (2010).

[4] H. M. Nguyen, O. Seitz, D. Aureau, A. Sra, N. Nijem, Y. N. Gartstein, Y. J. Chabal, A. V. Malko, Appl. Phys. Lett., 98, 161904, (2011).

[5] H. M. Nguyen, O. Seitz, W. Peng, Y. N. Gartstein, Y. J. Chabal, A. V. Malko, ACS Nano 6, 5574-5582 (2012).

[6] T. Franzl, T. A. Klar, S. Schietinger, A. L. Rogach and J. Feldmann, Nano Lett. 4, 1599 (2004).

[7] T. A. Klar, T. Franzl, A. L. Rogach and J. Feldmann, Adv. Mater. 17, 769 (2005).

[8] S. Nizamoğlu, P. L. Hernandez-Martinez, E. Mutlugün, D. U. Karatay and H. V. Demir, Appl. Phys. Lett. 100, 241109 (2012). 